Article

\title{
Model-Based Optimization of a Plug-In Hybrid Electric Powertrain with Multimode Transmission
}

\author{
Stefan Geng *, Andreas Meier and Thomas Schulte \\ Department 5-Electrical Engineering and Computer Science, Ostwestfalen-Lippe University of Applied \\ Sciences, Liebigstraße 87, 32657 Lemgo, Germany; andreas.meier@hs-owl.de (A.M.); \\ thomas.schulte@hs-owl.de (T.S.) \\ * Correspondence: stefan.geng@hs-owl.de
}

Received: 3 May 2018; Accepted: 7 June 2018; Published: 13 June 2018

\begin{abstract}
Plug-in hybrid electric vehicles are developed in order to reduce the fuel consumption and the emission of carbon dioxide. Besides the series, parallel and power split configurations are commonly used for conventional hybrid electric vehicles, and multimode transmissions are used for plug-in hybrid electric vehicles, which are able to switch between different modes like parallel or series operation of the combustion engine and electric motor. Several concepts have already been discussed and presented. These concepts comprise novel structures and multi-speed operation for the combustion engine and the electric motor, respectively. For improving the fuel and energy consumption, model-based optimizations of multimode transmissions are performed. In the first step of the optimization, the optimal number of gears and transmission ratios, as well as the corresponding fuel and energy savings, are estimated. Based on these results, a new multimode transmission concept with two-speed transmissions for the combustion engine and the electric motor has been developed. The knowledge of the concrete concept enables the further optimizations of the transmission ratios and the transmission control. In order to prove the benefit of the new and optimized transmission concept, powertrain simulations have been carried out. The new powertrain concept is compared to a powertrain concept with single-speed transmissions for the internal combustion engine (ICE) and electric motor operation. The new transmission concept enables a significant improvement of the fuel consumption.
\end{abstract}

Keywords: PHEV (plug-in hybrid electric vehicles), transmission; optimization; simulation; efficiency

\section{Introduction}

Today, vehicle manufacturers are forced to reduce the fuel consumption of their products because of enhanced environmental regulations. A promising solution is the development of plug-in hybrid electric vehicles (PHEV), as they combine an extended electric cruising range and the possibility to propel the vehicle by an internal combustion engine (ICE) when the battery is depleted or when high performance is required (e.g., on highways). Common powertrain configurations of PHEVs are the series-, parallel-, and power-split configurations. The efficiencies of these configurations varies, depending on the distance and the power demand of the trip that will be made [1]. For this reason, special transmission concepts have been developed, which enables the switching of the operation mode of the powertrain. Each operation mode conforms one of the common powertrain configurations and the capability of switching them during the vehicle operation will combine the advantages of the available powertrain configurations.

These so called multimode or dedicated hybrid transmissions (DHT) are optimized for the application in PHEVs. Compared to add-on solutions, where the electric motor is added to a conventional automatic transmission, DHTs are less complex (less speeds) and the electric motors 
are an integral part of the transmission. A simple and compact transmission design saves space and weight, which in turn, compensates a part of the additional space and weight of the electric motors and the battery. It also reduces the costs of the overall powertrain and makes PHEVs increasingly economic for customers. A general property of DHTs is that the electric motor is indispensable for the operation of the overall powertrain [2]. Some vehicle manufacturers already offer DHTs, (e.g., the Toyota with the 4th generation of the Prius [3], GM with the 'Voltec' system [4], or Mitsubishi with the multimode transmission [5]). Figure 1 shows the concept of the multimode transmission, where the electric motor and the ICE are connected to the final drive by means of fixed transmission ratios. Depending on the state of the clutch, the transmission enables a series hybrid mode, a parallel hybrid mode, an electric driving mode and an ICE mode. As a result of the fixed gear ratio, the ICE can be operated with a mechanical coupling to the final drive, only at relatively high vehicle speeds. At lower speeds, the vehicle can be driven by the electric motor, and the series hybrid mode is only necessary when the battery is depleted. Consequently, without the electric motor, the whole powertrain concept would be inoperative.

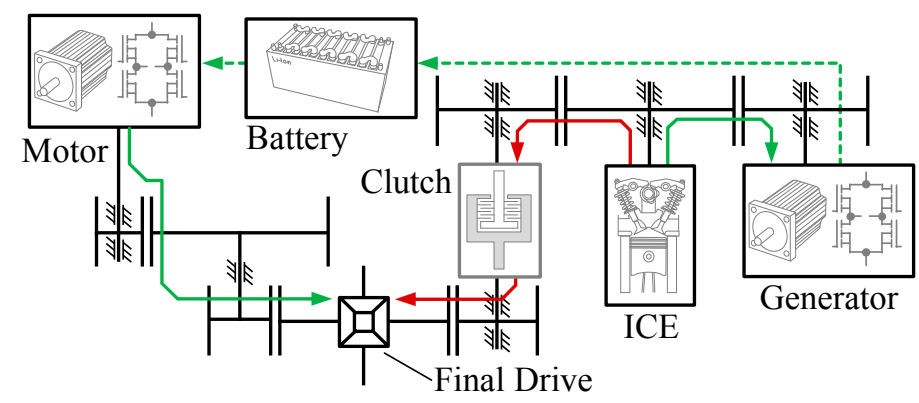

\begin{tabular}{l|c}
\multicolumn{1}{c|}{ Mode } & Clutch State \\
\hline series $=-$ & disengaged \\
electric & disengaged \\
driving & engaged \\
parallel $=$ & engat \\
ICE - & engaged
\end{tabular}

Figure 1. Multimode transmission with fixed gear ratios for the internal combustion engine (ICE) and electric motor.

The previous work [6] showed that a multi-speed transmission for the ICE and electric motor operation provides a significant potential for the reduction of fuel and energy consumptions. However, in this investigation, no operating strategy (combined operation of the ICE and electric motor) was considered. The optimization of the number of speeds and transmission ratios as well as the evaluation of fuel and energy consumptions were carried out separately. Based on this work, in [7] the evaluation, of fuel and energy consumptions was refined by applying dynamic programming (DP) as operating strategy. The obtained results were still promising, despite the fact that the optimization of the transmission was carried out without operating strategy.

In the work of Silvas et al. [8], an overview and general description of the methods for topology and component optimization, as well as for optimal control of hybrid electric vehicles is presented. An analysis of the yearly scientific publications showed that DP and genetic algorithms (GA) were increasingly used for powertrain optimization during the last few years. The optimization of gear ratios within a plug-in hybrid electric powertrain is discussed by the authors of [9], where a powertrain with two electric motors and two fixed gear ratios-one for each motor-is considered. DP is applied for powertrain control and the transmission ratios are determined by calculating the fuel consumptions for all possible transmission ratios and then the best one is chosen. In the work of [10], a GA is used to optimize the components of an electric vehicle with two electric motors and a dual multi-speed transmission. This method requires mathematical meta-models, which approximate the physical behavior of the system sufficiently. In terms of the transmission, the number of speeds and transmission ratios are considered for optimization. A similar approach is chosen in [11], where convex functions were used to approximate the engine's and motor's efficiency behavior. The advantage of having a convex optimization problem is that it can be solved analytically, which allows for finding the global optimum and enables fast calculations. The optimization goal is the operating strategy and the battery 
size of a city bus with a twelve-speed transmission. In the work of Kim et al. [12], an optimization of a PEHV's topology is presented. Here, mainly power-split configurations are optimized, where the corresponding model of the powers-split transmission considers gear friction, drag losses, and oil pump losses. DP is applied as an operating strategy in order to analyze the efficiency of different powertrain topologies.

In this contribution, a model-based development of a new DHT concept with multiple speeds is presented. As a general basis for the development, a vehicle similar to the Mitsubishi Outlander is considered. Unlike the powertrain concept of the Mitsubishi Outlander (see, Figure 1), the new concept will comprise only one electric motor [13]. In order to exploit the maximum efficiency of the transmission concept, a model-based optimization of the number of speeds and the related transmission ratios is carried out. Unlike in the previous works [6,7], the optimization of the transmission ratios will incorporate an operating strategy. The properties of all of the powertrain components (ICE, electric motor, battery, and vehicle dynamics) are chosen to be similar to the Mitsubishi Outlander, and they are not subject to the optimization.

The powertrain model and the considered powertrain components and its parametrization are presented in Section 2. Section 3 contains the optimization of the number of speeds and a first estimation of the transmission ratios. Based on these results, a new transmission concept was developed, which is presented in Section 4. As a result of the knowledge of the specific transmission configuration, a further optimization of the transmission ratios is carried out. The second optimization step requires an operation strategy, which defines the optimal control trajectory of the powertrain. A description of the method that is used to determine the optimal control trajectory is also contained in Section 5. Finally, the new transmission concept is evaluated by comparing the simulation results of the optimized concept with those of the concept that is shown in Figure 1.

\section{Powertrain Model}

An appropriate modelling approach for the purpose of optimization needs to combine the good adaptability of the system parameters and a low computational effort. This is obtained by the so-called backward approach [14], where beginning with the requested acceleration $a_{\mathrm{veh}}$ and speed $v_{\text {veh }}$ of the vehicle, the operating states of each powertrain component are determined backwards, see Figure 2. It is always assumed that the ICE and the electric motors are capable of generating the required torque and satisfying the requested vehicle acceleration, respectively. The submodels of the powertrain components consider the stationary states only, which is why no controllers for the powertrain components are required. Nevertheless, since a PHEV is considered, the operation mode for the powertrain must be controlled. The operation mode $s_{\mathrm{m}}$ is set by the operating strategy, which controls the transmission and also the ICE or electric motor. For example, in a parallel hybrid mode, the torque-split between the ICE and electric motor needs to be controlled (output $\widetilde{\mathbf{u}}$, in Figure 2). Since no dynamic behavior is considered, the operation mode changes immediately without any transition (e.g., continuously changing the state of a clutch from engaged to disengaged). The output of the powertrain simulation is the mass flow rate of the fuel $m_{\mathrm{f}}$ and the state of charge of the battery SoC.

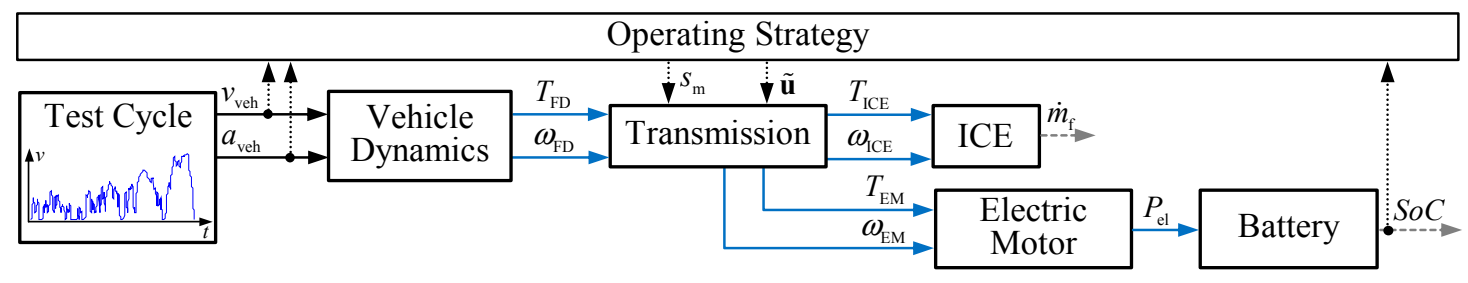

Figure 2. Powertrain model according to the backward approach. 
Figure 3 shows the submodels of the powertrain and their most significant parameters. The vehicle dynamics are considered in a longitudinal direction only, where the final drive torque $T_{\mathrm{FD}}$ is determined according to the following:

$$
T_{\mathrm{FD}}=r_{\mathrm{w}} \cdot\left(F_{\mathrm{drag}}\left(v_{\mathrm{veh}}\right)+F_{\mathrm{roll}}+F_{\mathrm{downhill}}+m_{\mathrm{veh}} \cdot a_{\mathrm{veh}}\right)
$$

with the radius of the wheels $r_{\mathrm{W}}$, the vehicle mass $m_{\mathrm{veh}}$, the vehicle speed $v_{\mathrm{veh}}$, and the vehicle acceleration $a_{\mathrm{veh}}$ (extracted from the test cycle).

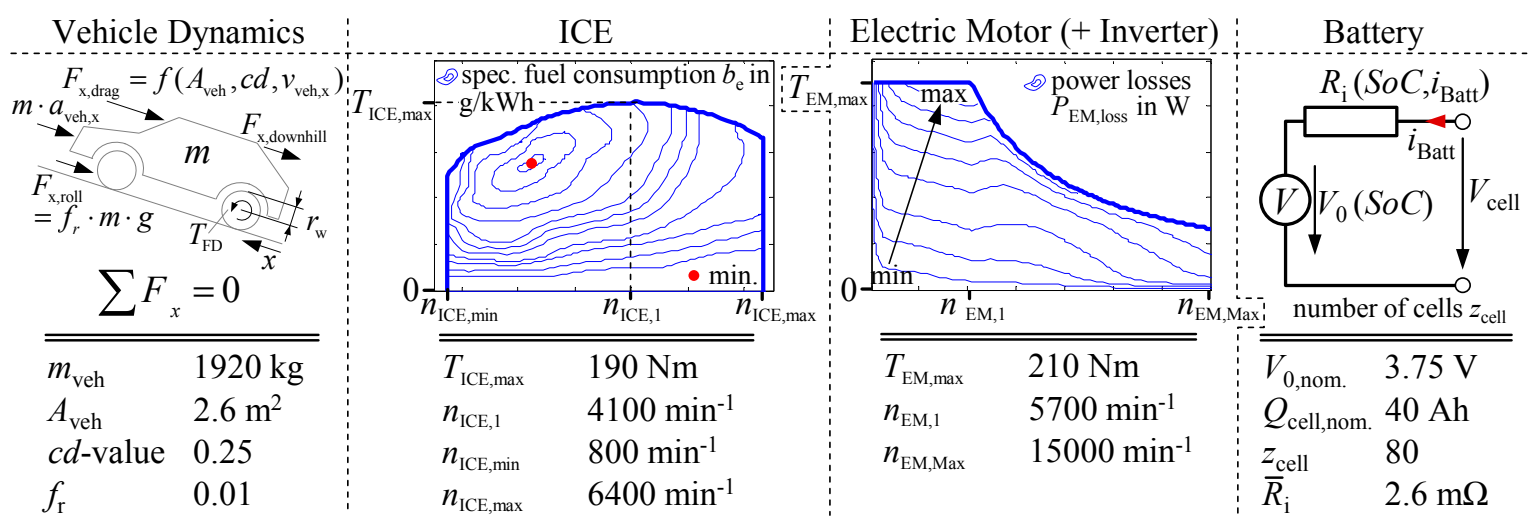

Figure 3. Models of the powertrain components and the most significant parameters.

The forces in Equation (1) represent the driving resistances comprising the drag force $F_{\mathrm{drag}}$, the rolling friction force $F_{\text {roll }}$, and the downhill force $F_{\text {downhill. }}$. The torque $T_{\mathrm{FD}}$ and the rotational speed $\omega_{\mathrm{FD}}$ at the final drive are required so as to evaluate a transmission model, which determines the torques and rotational speeds of the ICE and the electric motor, by considering the operation mode $s_{\mathrm{m}}$ and the corresponding transmission ratios. An automatic model generation is used to generate the a transmission model, $\mathrm{s}$ follows:

$$
\left[T_{\mathrm{ICE}} T_{\mathrm{EM}} \omega_{\mathrm{ICE}} \omega_{\mathrm{EM}}\right]^{T}=\mathbf{f}_{\mathrm{Trans}}\left(T_{\mathrm{FD}}, \omega_{\mathrm{FD}}, s_{\mathrm{m}}, \widetilde{u}\right)
$$

where the degree of detail (e.g., transmission dynamics, drag, and friction losses, etc.) is adjustable (see $[6,15])$. In this contribution, Equation (2) is a stationary function $\mathbf{f}_{\text {Trans, }}$, which represents a matrix multiplication of a coefficient matrix containing the corresponding transmission ratios and an input vector containing all of the necessary torques and rotational speeds. For each operation mode $s_{\mathrm{m}}$, a specific coefficient matrix is applied and an additional input $\widetilde{u}$ containing the desired torque or rotational speed for the electric motor is considered (only when necessary, e.g., torque-split in parallel hybrid mode).

The ICE is represented by a map, which describes the specific fuel consumption $b_{\mathrm{e}}$ as a function

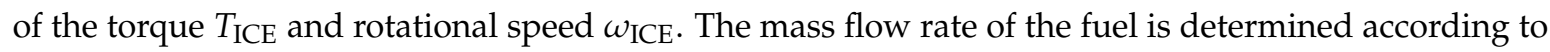
the following:

$$
\dot{m}_{\mathrm{f}}=\frac{b_{\mathrm{e}}\left(T_{\mathrm{ICE}}, \omega_{\mathrm{ICE}}\right) \cdot T_{\mathrm{ICE}} \cdot \omega_{\mathrm{ICE}}}{3.6 \cdot 10^{6}}
$$

with $m_{\mathrm{f}}$ in $g \cdot s^{-1}$. Similarly, the required electric power $P_{\mathrm{el}}$ of the electric motor is determined, as follows:

$$
P_{\mathrm{el}}=T_{\mathrm{EM}} \cdot \omega_{\mathrm{EM}}+P_{\mathrm{EM}, \mathrm{loss}}\left(\left|T_{\mathrm{EM}}\right|,\left|\omega_{\mathrm{EM}}\right|\right)
$$

where the power loss $P_{\mathrm{EM}, \text { loss }}$ is a result of the map, shown in Figure 3. This map also includes the power losses of the inverter. Since the electric power $P_{\mathrm{el}}$ must be provided by the battery, a battery current will be caused, as follows: 


$$
\dot{q}=i_{\text {Batt }}=-\frac{V_{0}(S o C)}{R_{\mathrm{i}}(S o C)}+\sqrt{\left(\frac{V_{0}(S o C)}{R_{\mathrm{i}}(S o C)}\right)^{2}-\frac{P_{\mathrm{el}}}{z_{\text {cell }}}} .
$$

Equation (5) follows from the simplified equivalent circuit in Figure 3, where $z_{\text {cell }}$ is the number of cells, $V_{0}$ the internal cell voltage, and $R_{\mathrm{i}}$ the internal cell resistance.

In this representation $V_{0}$ and $R_{\mathrm{i}}$ are a function of the state of charge only, as follows:

$$
\text { SoC }=\frac{100}{3600} \cdot \int \frac{i_{\text {Batt }}}{z_{\text {cell }} \cdot Q_{\text {cell,nom }}} d t
$$

which is determined by integrating Equation (5) and dividing the result by the nominal battery capacity $Q_{\text {cell,nom. }}$. Since the powertrain model is executed in discrete time steps, Equation (5) can be solved explicitly (no algebraic loop occur).

For the optimization in the following chapter, the powertrain model that has been described above is used and parameterized according to Figure 3. The second optimization step in Section 5 requires a convex powertrain model, which approximates the model from above by means of parabolic functions. Because the transmission concept is refined in each optimization step, several transmission models with a different degree of detail are used. A short description of the used transmission model is given in the corresponding chapters.

\section{Optimization: Number of Speeds}

In this chapter, the impact of the number of speeds for the ICE and electric motor operation on the fuel and energy consumption is investigated. Since no specific transmission concept is known within this stage of the development, a simplified transmission model is used. It represents two separate transmission ratios, one for the ICE and one for the electric motor. Both of the transmission ratios can be switched during the simulation in order to represent multi-speed transmissions.

For this investigation, the operation of the ICE and the electric motor are considered separately, that is, the energy for propelling the vehicle comes either from the fuel or the battery. It is intended to analyze the benefit of multiple speeds for each power source and to estimate the optimal number of the speeds. Furthermore, it is assumed that the impact of multiple speeds on the combined operation of the ICE and electric motor is comparable to that of the separated consideration. If only one power source is considered, the optimal shift strategy (choice of the optimal gear $s_{\mathrm{g}}$ ) can be determined without considering any specific test cycle. Therefore, the optimal choice of the gear $s_{\mathrm{g}}$ is determined according to the following:

$$
P_{\text {loss }}=\left(v_{\mathrm{veh}}, T_{\mathrm{FD}}, s_{\mathrm{g}}^{*}\right)=\min _{\mathrm{sg}_{\mathrm{g}} \in \mathbb{N}} P_{\text {loss }}\left(v_{\mathrm{veh}}, T_{\mathrm{FD}}, s_{\mathrm{g}}\right)
$$

where the overall power loss within the powertrain $P_{\text {loss }}$ is minimized for all of the possible final drive torques $T_{\mathrm{FD}}$ and vehicle speeds $v_{\mathrm{veh}}$. As a result, a map that defines the optimal choice of the gear $s_{\mathrm{g}}^{*}\left(T_{\mathrm{FD}}, v_{\mathrm{veh}}\right)$ is obtained. Since the number of possible solutions for Equation (7) is equal to the number of speeds, a feasible and still fast method for solving Equation (7) is to check all of the possibilities and to choose the best one.

Figure 4a shows the procedure for optimizing the transmission ratios of a multi-speed transmission in terms of a minimal fuel and energy consumption, respectively. The simplex method is applied to find the optimal set of transmission ratios $\mathbf{i}_{\mathrm{ICE}, \mathrm{EM}}^{*}$, which causes the lowest consumption for a given test cycle. For each set of $\mathbf{i}_{\mathrm{ICE}, \mathrm{EM}}$, the shift strategy is adapted and the powertrain model is executed, where the resulting fuel consumption $V_{\text {fuel }}$ or energy consumption $E$ represents the cost function for the optimization. In order to consider a wide range of vehicle speeds and accelerations, the test cycles WLTP (Worldwide Harmonized Light Vehicles Test Procedure), Urban Dynamometer, and FTP 75 are considered. A scalar cost function is obtained by averaging the results of these three test 
cycles. Figure $4 \mathrm{~b}$ shows the energy savings, which can be obtained by multi-speed transmissions for the electric motor operation. The diagram shows the energy savings as a function of the transmission ratios for a two-speed transmission, and the table contains the optimized energy consumptions and the corresponding transmission ratios for a one- to four-speed transmission. In order to ensure that the electric motor can be operated up to a vehicle speed of $180 \mathrm{~km} / \mathrm{h}$, the transmission ratio of the highest gear is constrained to values smaller than 10.37. The table shows that the benefit of a two-speed transmission is up to $3.5 \%$ energy saving, whereas the benefit of each additional speed becomes drastically smaller.

a)

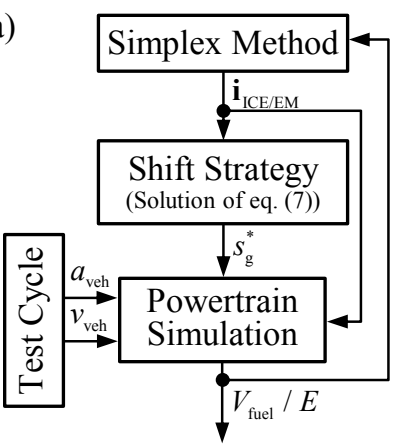

b)

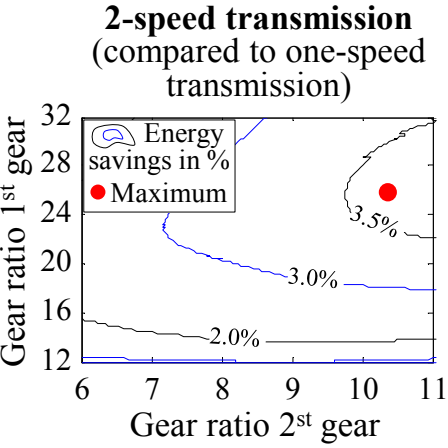

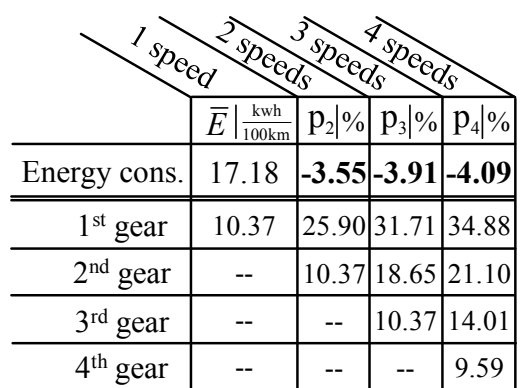

Figure 4. (a) Optimization procedure of the transmission ratios in terms of a minimal fuel and energy consumption and (b) optimized energy savings and transmission ratios of a multi-speed transmissions for the electric motor operation.

The fuel savings, which can be obtained by multi-speed transmissions for the ICE operation, are shown in Figure 5, where the diagrams depict the functions of fuel saving for a two- and three-speed transmission. In order to enable a maximum vehicle speed of $180 \mathrm{~km} / \mathrm{h}$, the transmission ratio of the highest gear is always 3.47. Since not all of the operating conditions of the vehicle can be driven by the ICE, an imaginary series hybrid mode is introduced, where the ICE is decoupled from the final drive and operated according to the characteristic of minimal fuel consumption. It is assumed that the ICE must produce $30 \%$ more mechanical power than what is required at the final drive. The table in Figure 5 shows that, compared to a one-speed transmission, the benefit of a two-speed transmission is an up to $8.1 \%$ fuel saving and of a 3 speeds transmission of up to $9.5 \%$. Each additional speed increases the fuel saving, but the benefit compared to a lower number of speeds decreases.
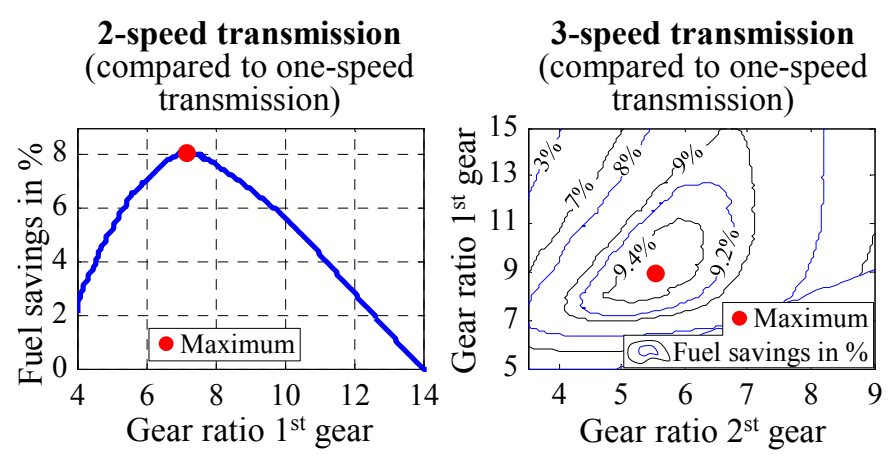

\begin{tabular}{c|c|c|c|c|c|c|}
\hline & $\bar{V} \mid \frac{1}{100 \mathrm{~km}}$ & $\mathrm{p}_{2} \mid \%$ & $\mathrm{p}_{3} \mid \%$ & $\mathrm{p}_{4} \mid \%$ & $\mathrm{p}_{5} \mid \%$ & $\mathrm{p}_{6} \mid \%$ \\
\hline Fuel cons. & 6.2 & $\mathbf{- 8 . 1}$ & $\mathbf{- 9 . 5}$ & $\mathbf{- 1 0 . 1}$ & $\mathbf{- 1 0 . 5}$ & $\mathbf{- 1 0 . 6}$ \\
\hline \hline $1^{\text {st }}$ gear & 3.47 & 7.13 & 9.02 & 12.27 & 16.62 & 23.54 \\
\hline $2^{\text {nd }}$ gear & -- & 3.47 & 5.53 & 7.41 & 8.89 & 10.82 \\
\hline $3^{\text {rd }}$ gear & -- & -- & 3.47 & 5.10 & 6.23 & 7.54 \\
\hline $4^{\text {th }}$ gear & -- & -- & -- & 3.47 & 4.56 & 6.01 \\
\hline $5^{\text {th }}$ gear & -- & -- & -- & -- & 3.47 & 4.53 \\
\hline $6^{\text {th }}$ gear & -- & -- & -- & -- & -- & 3.47 \\
\hline
\end{tabular}

Figure 5. Optimization results of multi-speed transmissions for the ICE operation. Left and middle: visualization of the relation between transmission ratios and fuel savings. Right: optimized fuel savings and transmission ratios.

For the ICE and the electric motor, the most beneficial number of speeds is two. The additional benefit of every additional speed decreases and it must be taken into account that the transmission 
becomes more complex when the number of speeds rises. Because of a complex transmission, the power losses and weight will increase, which might diminish the small increment of fuel savings for an additional speed. It must also be considered that, because of the separate consideration of the ICE and the electric motor, the transmission ratios are an estimation only. More accurate results can be obtained by considering the combined operation of the ICE and electric motor. The results that are shown in Figures 4 and 5 are taken as a basis for the development of a new DHT concept and an additional optimization step, where the combined operation is considered.

\section{New Transmission Concept}

Based on the results of the optimizations in Section 3, the new transmission concept, depicted in Figure 6, was developed [13]. A two-speed transmission for the ICE and the electric motor operation is chosen, as it enables the biggest benefit in terms of fuel and energy savings, by means of the lowest complexity of the transmission. Because of the fact that at low speeds the vehicle can only be propelled with the electric motor, it must be ensured that, even if the battery is discharged, the operation of the vehicle is still possible. Therefore, the new concept enables a continuous variable transmission (CVT) mode. In this mode, the rotational speeds of the ICE and electric motor are superimposed by means of a planetary gear, which enables the control of the rotational speed of the ICE with the speed of the electric motor. The speed of the ICE can be adjusted continuously as a function of the speed of the electric motor and the final drive, see Equation (10). The design of the transmission ensures that the electric motor operates as a generator in CVT mode, until an appropriate vehicle speed is reached, where the ICE mode can be activated.

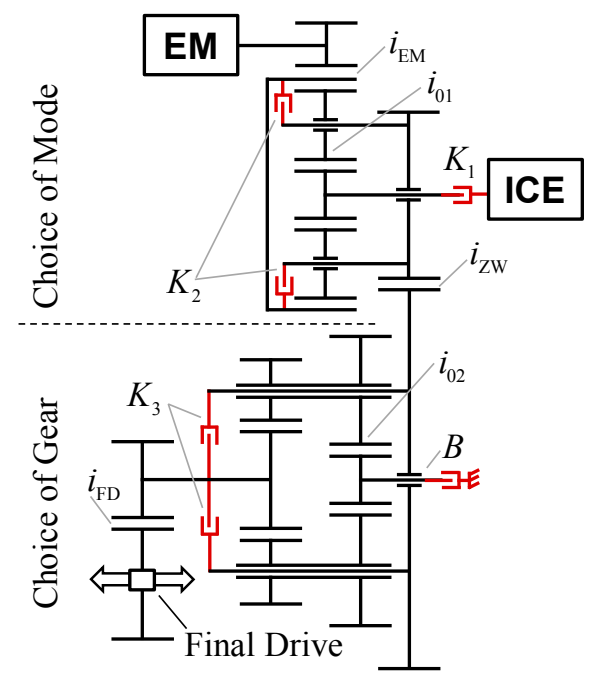

Modes: $\times$ Clutch or brake engaged
\begin{tabular}{c|c|c|c|c|c|c|} 
CVT1 & CVT2 & ICE1/ & ICE2/ & EM1 & EM2 \\
& $\left(s_{\mathrm{m}}=1\right)$ & $\left(s_{\mathrm{m}}=2\right)$ & $\begin{array}{c}\text { PAR } \\
\left(s_{\mathrm{m}}=3\right)\end{array}$ & $\begin{array}{c}\text { PAR } \\
\left(s_{\mathrm{m}}=4\right)\end{array}$ & $\left(s_{\mathrm{m}}=5\right)$ & $\left(s_{\mathrm{m}}=6\right)$ \\
\hline Clutch $K_{1}$ & $\times$ & $\times$ & $\times$ & $\times$ & & \\
\hline Dog Clutch $K_{2}$ & & & $\times$ & $\times$ & $\times$ & $\times$ \\
\hline Clutch $K_{3}$ & & $\times$ & & $\times$ & & $\times$ \\
\hline Brake $B$ & $\times$ & & $\times$ & & $\times$ & \\
\hline
\end{tabular}

Figure 6. New transmission concept and the switching pattern of the clutches and brakes for activating the available operation modes.

According to Figure 6, the first planetary gear is used to change the operation mode and the second planetary gear (epicyclic gear with two sun gears) is used to change the gear (two-speed transmission). If the dog clutch $K_{2}$ is disengaged, the rotational speeds of the ICE and electric motor are superimposed and the powertrain operates in CVT-mode. Otherwise, if $K_{2}$ is engaged, the planets are blocked and the whole planetary gear rotates as one common body. In this case, the electric driving mode, the ICE mode, or the parallel hybrid mode can be active. When the ICE is used for propulsion, the clutch $K_{1}$ must be engaged. The brake $B$ activates the first gear and the multi-plate clutch $K_{3}$ activates the second gear. Each operation mode can be driven in two different speeds. 
The overall transmission ratio of the ICE mode is as follows:

$$
i_{\mathrm{ICE}, \mathrm{FD}}=\frac{-T_{\mathrm{FD}, \mathrm{ICE}}}{T_{\mathrm{ICE}}}=\frac{\omega_{\mathrm{ICE}}}{\omega_{\mathrm{FD}}}=\widetilde{i} \cdot i_{\mathrm{PG} 2}
$$

and of the electric motor mode is as follows:

$$
i_{\mathrm{EM}, \mathrm{FD}}=\frac{T_{\mathrm{FD}, \mathrm{EM}}}{T_{E M}}=\frac{-\omega_{\mathrm{EM}}}{\omega_{\mathrm{FD}}}=i_{\mathrm{EM}} \cdot \tilde{i} \cdot i_{\mathrm{PG} 2}
$$

with $\widetilde{i}=i_{\mathrm{FD}} \cdot i_{\mathrm{ZW}}$ and $i_{\mathrm{PG} 2}=1 /\left(1-i_{02}\right)$ for the first gear and $i_{\mathrm{PG} 2}=1$ for the second gear. The final drive torque $T_{\mathrm{FD}}$ will be composed of the sum of $T_{\mathrm{FD}, \mathrm{ICE}}$ and $T_{\mathrm{FD}, \mathrm{EM}}$.

Considering the CVT mode, the overall transmission ratio regarding the rotational speed $\omega_{\text {ICE }}$ of the ICE is a function of the rotational speed of the electric motor $\omega_{\mathrm{EM}}$ and the final drive $\omega_{\mathrm{FD}}$, as follows:

$$
i_{\omega, \mathrm{CVT}, \mathrm{FD}}=\frac{\omega_{\mathrm{ICE}}}{\omega_{\mathrm{FD}}}=\frac{i_{01}}{i_{\mathrm{EM}}} \frac{\omega_{\mathrm{EM}}}{\omega_{\mathrm{FD}}}+\left(1+i_{01}\right) \cdot \widetilde{i} \cdot i_{\mathrm{PG} 2}
$$

which enables an adjustment of the rotational speed of ICE $\omega_{\mathrm{ICE}}$ for a given final drive speed $\omega_{\mathrm{FD}}$, by means of $\omega_{\mathrm{EM}}$. According to the transmission ratio regarding the torques, as follows:

$$
i_{\mathrm{T}, \mathrm{CVT}, \mathrm{FD}}=\frac{-T_{\mathrm{FD}}}{T_{\mathrm{ICE}}}=\frac{i_{01} \cdot T_{\mathrm{FD}}}{i_{\mathrm{EM}} \cdot T_{\mathrm{EM}}}=\left(1+i_{01}\right) \cdot \widetilde{i} \cdot i_{\mathrm{PG} 2}
$$

the torques of the ICE $T_{\mathrm{ICE}}$ and the electric motor $T_{\mathrm{EM}}$ must fulfill the torque equilibrium of the planetary gear.

\section{Optimization: Transmission Ratios}

In order to determine the optimal transmission ratios of the new transmission concept, a further optimization step is carried out. The transmission model that is used for the optimization considers the concept in Figure 6, where the torques and angular speeds are calculated according to the transmission ratios of the planetary and spur gears. As an initial parameterization, the transmission ratios that are obtained in Section 3 are used. For the optimization, the minimal fuel consumption for a given set of transmission ratios and test cycles has to be calculated. This can only be enabled if the combined operation of the ICE and the electric motor is considered (ICE, Parallel, or CVT-mode) and controlled optimally. Generally, an optimal control trajectory for the powertrain $\mathbf{u}^{*}(t)$ must be determined, which solves the optimization problem as follows:

$$
\begin{gathered}
\min _{\mathbf{u}(t)}\left\{J=\int_{0}^{t_{\mathrm{e}}} \dot{m}_{\mathrm{f}}\left(T_{\mathrm{ICE}}, \omega_{\mathrm{ICE}}\right) d t\right\} \\
\dot{q}=f_{\mathrm{Batt}}\left(P_{\mathrm{el}}\left(T_{\mathrm{EM}}, \omega_{\mathrm{EM}}\right)\right) \\
{\left[T_{\mathrm{ICE}} T_{E M} \omega_{\mathrm{ICE}} \omega_{E M}\right]^{T}=\mathbf{f}_{\mathrm{Trans}}\left(T_{\mathrm{FD}}, \omega_{\mathrm{FD}}, \mathbf{u}\right)} \\
q \in \mathcal{X}, \mathbf{u}=\left[s_{\mathrm{m}} \widetilde{u}\right]^{T} \in \mathcal{U}^{2}
\end{gathered}
$$

with the cost function $J$ and the duration of the test cycle $t_{\mathrm{e}}$. The control vector $\mathbf{u}$ summarizes the operation mode $s_{\mathrm{m}}$ and the variable control input for the electric motor, as follows:

$$
\widetilde{u}:= \begin{cases}\widetilde{\omega}_{\mathrm{EM}} & , s_{\mathrm{m}}=1 \vee 2 \\ \widetilde{T}_{\mathrm{EM}} & , s_{\mathrm{m}}=3 \vee 4 \\ 0 & , s_{\mathrm{m}}=5 \vee 6\end{cases}
$$


where the allocation of the shown numbers is found in Figure 6. The optimal control trajectory $\mathbf{u}^{*}(t)$ will satisfy the state equation $q$ as well as the state space constraints $\mathcal{X}$ and control space constraints $\mathcal{U}^{2}$. In this contribution, a combination of the optimization methods dynamic programming (DP) and calculus of variations $(\mathrm{CoV})$ is applied, where DP determines the discrete input $s_{\mathrm{m}}$ and $\mathrm{CoV}$ the continuous input $\widetilde{u}$. This method was already presented by the authors of [16] and enables time-efficient optimizations. However, since the method was applied on a parallel hybrid vehicle with a six-speed transmission for the ICE, it had to be adapted to the new more complex vehicle configuration. In the next sections, the basics of $\mathrm{CoV}$ and DP and the combination of both are described. Finally, the results that were obtained by applying the proposed method for the optimization of the transmission ratios are presented.

\subsection{Calculus of Variations (CoV)}

The basics of the $\mathrm{CoV}$ are presented by the authors of [17]. The optimal continuous input $\widetilde{u}^{*}$ is obtained by minimizing the Hamiltonian function, as follows:

$$
\mathcal{H}=\dot{m}_{\mathrm{f}}\left(T_{\mathrm{FD}}, \omega_{\mathrm{FD}}, s_{\mathrm{m}}, \widetilde{u}\right)+\lambda \cdot \dot{q}\left(T_{\mathrm{FD}}, \omega_{\mathrm{FD}}, s_{\mathrm{m}}, \widetilde{u}\right)
$$

in terms of the input $\widetilde{u}$. It is assumed that the Lagrange multiplier $\lambda$ is known in advance and that the optimal solution $\widetilde{u}^{*}$ does not have to be bounded to the limits of the control space constraint $\mathcal{U}$. Furthermore, the final drive torque $T_{\mathrm{FD}}$ and angular velocity $\omega_{\mathrm{FD}}$, as well as the operation mode $s_{\mathrm{m}}$, are considered here as constants. Then, a potential minima of Equation (14) is obtained by fulfilling the necessary conditions, as follows:

$$
\begin{gathered}
\frac{\partial \mathcal{H}}{\partial \widetilde{u}}=0 \Leftrightarrow \widetilde{u}=f_{\widetilde{u}}(\lambda) \\
-\frac{\partial \mathcal{H}}{\partial q}=\dot{\lambda}
\end{gathered}
$$

where Equation (15) is rearranged according to $\widetilde{u}$. In this case, since the Lagrange multiplier $\lambda$ is assumed to be known, the second condition, Equation (16), is not required and the optimal solution $\widetilde{u}^{*}$ can be calculated directly by means of Equation (15). However, it must be taken into account that Equation (15) is only the optimal solution $\widetilde{u}^{*}$ if it also fulfills the sufficient condition for an extrema. According to the authors of [18], the sufficient condition is always fulfilled when the cost function $J$ is convex regarding $\widetilde{u}$. In order to obtain a convex cost function, the original data map of the fuel flow rate of the ICE (see Figure 3) is approximated by the parabolic function, as follows:

$$
\dot{m}_{\mathrm{f}, \mathrm{convex}}\left(T_{\mathrm{ICE}}, \omega_{\mathrm{ICE}}\right)=a_{2}\left(\omega_{\mathrm{ICE}}\right) \cdot T_{\text {ICE }}^{2}+a_{1}\left(\omega_{\mathrm{ICE}}\right) \cdot T_{\mathrm{ICE}}+a_{0}\left(\omega_{\mathrm{ICE}}\right)
$$

where the coefficients are a function of the rotational speed. It is required that Equation (17) is a convex function of $T_{\mathrm{ICE}}$, because it is directly affected by the input $\widetilde{u}$. In order to have an analytic equation for the Hamiltonian function, Equation (14), the power losses of the electric motor are also approximated by a convex function, as follows:

$$
P_{\mathrm{EM}, \text { loss,convex }}\left(T_{\mathrm{EM}}, n_{\mathrm{EM}}\right)=b_{2}\left(\omega_{\mathrm{EM}}\right) \cdot T_{\mathrm{EM}}^{2}+b_{1}\left(\omega_{\mathrm{EM}}\right) \cdot T_{\mathrm{EM}}+b_{0}\left(\omega_{\mathrm{EM}}\right)
$$

A further approximation is made, by using the convex state equation, as follows:

$$
\dot{q}_{\text {convex }}=i_{\text {Batt }}\left(P_{\mathrm{el}}\right)=c_{2} \cdot P_{\mathrm{el}}^{2}+c_{1} \cdot P_{\mathrm{el}}+c_{0}
$$

where the influence of the $S o C$ on the battery current $i_{\text {Batt }}$ is neglected. Considering Equations (17)-(19), the Hamiltonian function, Equation (14), becomes independent of $q$ and the derivation of $\lambda$ in Equation (16) becomes zero. This means that the Lagrange multiplier $\lambda$ must be a constant value. 


\subsection{Dynamic Programming (DP)}

The general application of the DP method is presented in the work of [19]. For the intended application, the Hamiltonian function is considered as cost function as follows:

$$
\min _{u_{\mathrm{m}}(t)}\left\{J_{\mathrm{DP}}=\int_{0}^{t_{\mathrm{e}}} \mathcal{H}\left(T_{\mathrm{FD}}, \omega_{\mathrm{FD}}, \lambda, s_{\mathrm{m}}\right) d t\right\}
$$

and the operation mode as a state variable, as follows:

$$
s_{\mathrm{m}, \mathrm{k}+1}=s_{\mathrm{m}, \mathrm{k}}+u_{\mathrm{m}, \mathrm{k}}
$$

with the discrete control input $u_{\mathrm{m}, \mathrm{k}} \in \mathbb{N} \subseteq\{-5, \ldots, 5\}$. It is still assumed that the constant Lagrange multiplier $\lambda$ is known in advance. Then, the Hamiltonian function can be evaluated for a given operation mode $s_{\mathrm{m}}$ by means of the CoV—Equations (14) and (15)—and the DP algorithm can determine a trajectory for the operation mode $s_{\mathrm{m}}$, which yields a minimal Hamiltonian function $\mathcal{H}$. The optimization problem is considered as time-discrete, where for each time step $t_{\mathrm{k}}=k \cdot T$ the local optimization problem is solved as follows:

$$
\mathbf{J}_{\mathrm{DP}, \mathrm{k}}\left(\mathbf{s}_{\mathrm{m}, \mathrm{k}}\right)=\min _{\mathbf{u}_{\mathrm{m}}}\left\{\mathbf{J}_{\mathrm{DP}, \mathrm{k}+1}\left(\mathbf{s}_{\mathrm{m}, \mathrm{k}+1}\right)+\mathbf{H}\left(T_{\mathrm{FD}, \mathrm{k}}, \omega_{\mathrm{FD}, \mathrm{k}}, \lambda, \mathbf{s}_{\mathrm{m}, \mathrm{k}}\right) \cdot T\right\}
$$

with the matrix $\mathbf{H}$ containing the Hamiltonian function values for all of the possible states $\mathbf{s}_{\mathrm{m}}$ and inputs $\mathbf{u}_{\mathrm{m}}$. Equation (22) is solved backwards in time from $k=N-1$ to $k=0$, with the initial costs $\mathbf{J}_{\mathrm{DP}, \mathrm{N}}=0$. The result in each time step $k$ is an optimal control vector $\mathbf{u}_{\mathrm{m}, \mathrm{k}}^{*}$ and the minimal accumulated costs $\mathbf{J}_{\mathrm{DP}, \mathrm{k}}$ for each state within the state grid vector $\mathbf{s}_{\mathrm{m}, \mathrm{k}}$. The optimal inputs $\mathbf{u}_{\mathrm{m}, \mathrm{k}}^{*}$ are also allocated to the state grid vector and are saved in a new matrix, as follows:

$$
\mathbf{U}_{\mathrm{m}, \mathrm{k}}^{*}\left(\mathrm{~s}_{\mathrm{m}, \mathrm{k}}^{*}\right)=\mathbf{u}_{\mathrm{m}, \mathrm{k}}^{*}
$$

After completing the backward calculation, the optimal control input trajectory $u_{\mathrm{m}}^{*}$ is determined by extracting the optimal inputs $u_{\mathrm{m}}^{*}$ from the matrix, Equation (23), according to the current operation mode $s_{\mathrm{m}, \mathrm{k}}$, as follows:

$$
u_{\mathrm{m}, \mathrm{k}}^{*}=\mathbf{U}_{\mathrm{m}, \mathrm{k}}\left(s_{\mathrm{m}, \mathrm{k}}\right) \text {. }
$$

Equation (24) is processed forward in time from $k=0$ to $k=N-1$, where the next operation mode $s_{\mathrm{m}, \mathrm{k}+1}$ is calculated according to Equation (21) by means of the result of Equation (24).

\subsection{DP and CoV Combined}

Figure $7 \mathrm{a}$ shows the algorithm for the optimization of the vehicle operation by means of the combination of DP and CoV. The constant Lagrange multiplier $\lambda$ is still unknown and must be determined. According to Figure $7 \mathrm{c}$, it affects the initial state of charge $S o C_{0}$ and only the correct $\lambda$ will satisfy the desired initial condition $S_{o} C_{0}^{*}$. The solution is determined by means of the Regula Falsi method, where the whole algorithm comprising DP and CoV is executed within each iteration step, until the solution $S o C_{0}^{*}$ is found.

The algorithm, which combines DP and $\mathrm{CoV}$, consists basically of the backward calculation of DP. It begins at $k=N-1$ and the first step is to apply the $\mathrm{CoV}$ in order to determine the Hamiltonian functions $\mathbf{H}$ for all of the operation modes of the state grid $\mathbf{s}_{\mathrm{m}, \mathrm{k}}$ and the current final drive torque $T_{\mathrm{FD}, \mathrm{k}}$ and speed $\omega_{\mathrm{FD}, \mathrm{k}}$. In the second step, all of the possible state transitions from $\mathbf{s}_{\mathrm{m}, \mathrm{k}}$ to $\mathbf{s}_{\mathrm{m}, \mathrm{k}+1}$ are calculated. Figure $7 \mathrm{~b}$ illustrates the state transitions from $t_{\mathrm{k}+1}$ to $t_{\mathrm{k}+2}$ for the initial state $s_{\mathrm{m}, \mathrm{k}}=6$ (grey arrows in upper part). Each transition corresponds to one input in $\mathbf{u}_{\mathrm{m}, \mathrm{k}}$, where the input, which causes less cos, is chosen as the optimal input (black arrow in upper part of Figure $7 \mathrm{~b}$ ). The choice of the optimal input is made in the third step, by minimizing the accumulated costs $\mathbf{J}_{\mathrm{DP}, \mathrm{k}}$. The result is 
the optimal control vector $\mathbf{u}_{\mathrm{m}, \mathrm{k}}^{*}$, which contains the optimal input for each operation mode of the state grid $\mathbf{s}_{\mathrm{m}, \mathrm{k}}$. The optimal input control for the operation states $\mathbf{u}_{\mathrm{m}, \mathrm{k}}^{*}$ and the electric motor $\widetilde{\mathbf{u}}_{\mathrm{k}}^{*}$ are saved in the fifth step. In order to evaluate the current $S o C_{k}$, the electric charge for all of the combinations of the states $\mathbf{s}_{\mathrm{m}, \mathrm{k}}$ and inputs $\mathbf{u}_{\mathrm{m}, \mathrm{k}}$ is determined and saved in $\mathbf{Q}_{\mathrm{k}}$ (step four). At the bottom of Figure $7 \mathrm{~b}$, an exemplary characteristic of the $\mathrm{SoC}$ is shown. Generally, more than one characteristic will exist and for each characteristic and time step, the optimal transition must be determined. This is solved by extracting the solutions, which belong to the optimal control vector $\mathbf{u}_{\mathrm{m}, \mathrm{k}^{\prime}}^{*}$ see step six in Figure $7 \mathrm{a}$.

a)

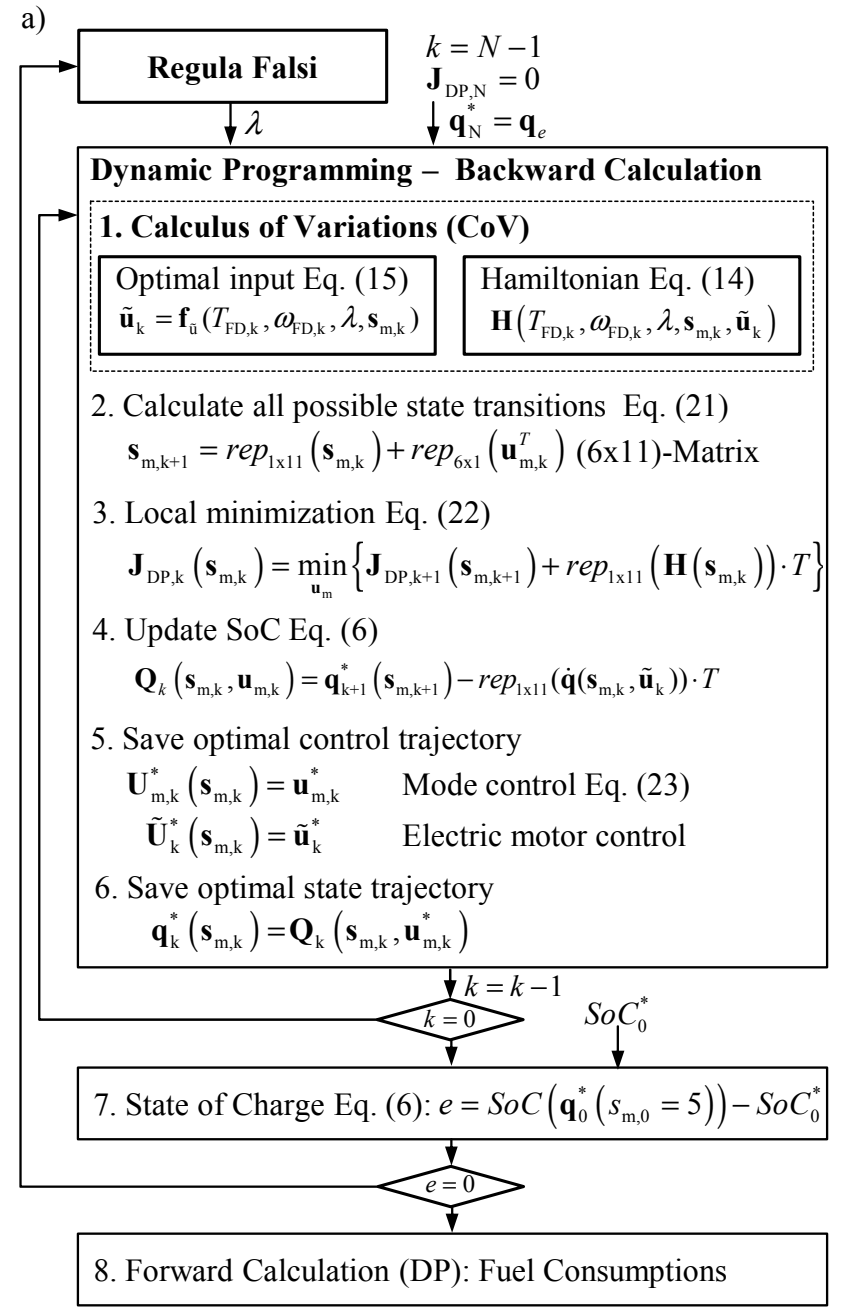

b)

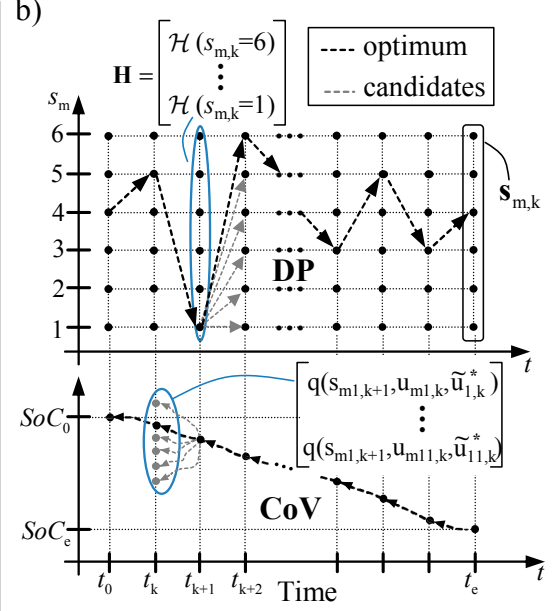

c)
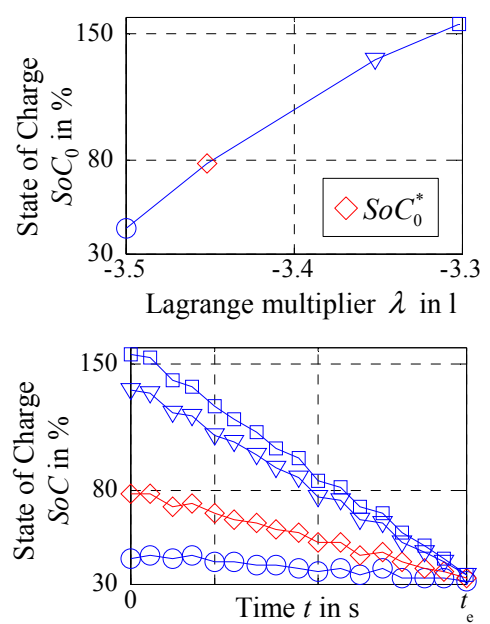

Figure 7. (a) Dynamic programming (DP) and calculus of variations (CoV) algorithm for the optimization of the plug-in hybrid electric vehicles (PHEV) operation; (b) schematic representation of the algorithm; and (c) example of the relation between the constant Lagrange multiplier $\lambda$ and the initial state $\mathrm{SoC}_{0}$ (above) and optimal state trajectory for a given $\lambda$ (below).

After completing the backward calculation, $S_{o} C_{0}$ is determined in step seven (electric driving is the initial operation state $S_{\mathrm{m}, 0}=5$ ). If the deviation regarding the desired value $S o C_{0}^{*}$ becomes small enough, the resulting fuel consumption is calculated in step eight by means of the forward calculation and the matrices that were saved in step five.

\subsection{Optimization Results}

The algorithm that is shown in Figure 7 enables the determination of the fuel consumption for a given set of test cycles. For the optimization of the transmission ratios of the new transmission 
concept, the test cycles FTP 75, Urban Dynamometer, and WLTP are considered. All of the test cycles are repeated until the overall distance is equal to approximately $200 \mathrm{~km}$ (ensures that the battery is discharged). The operation of the powertrain is optimized for the considered test cycles and a predefined range of the transmission ratios of the first ICE-mode and the second electric driving mode. As a result of the properties of the new transmission concept, all of the other transmissions ratios are defined by means of those two modes. Figure 8 shows the fuel savings as a function of the transmission ratios. It is compared to the fuel saving of the new transmission concept, which was parametrized according to the results that were obtained in Section 3. It turned out, that because of the additional optimization of the transmission ratios, a fuel saving of up to $1.4 \%$ is obtained compared to the parametrization, according to the separated consideration of ICE and electric motor in Section 3.

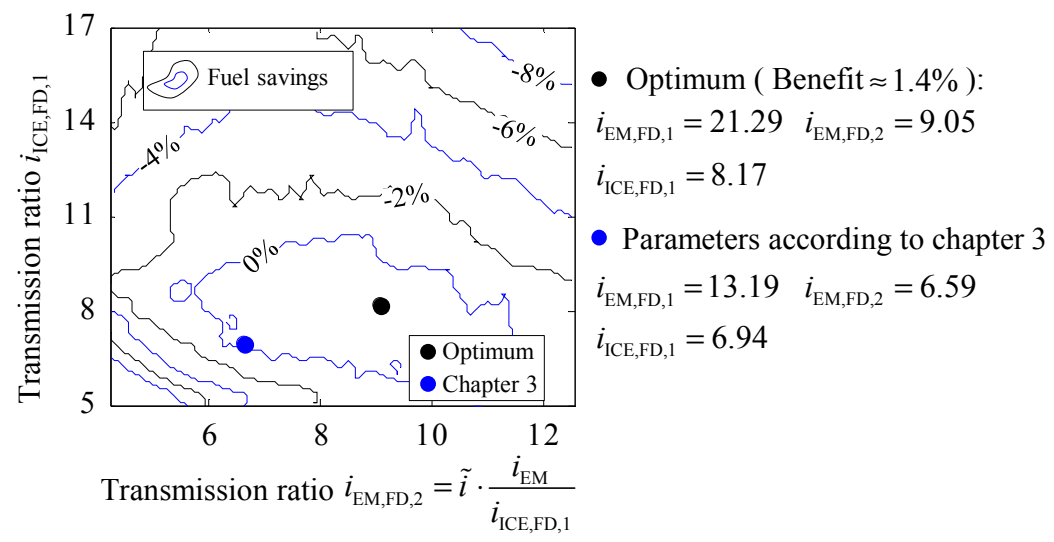

Figure 8. Visualization of the fuel savings achieved with the new transmission concept in dependency of the transmission ratios. For comparison, the average value fuel consumption of the three above mentioned test cycles is considered and as reference $(0 \%)$, the fuel consumption obtained with the parameters in Section 3, is chosen. The black dot indicates the optimum with approximately $1.4 \%$ benefit, compared to the parametrization in Section 3.

\section{Concept Evaluation}

A model-based evaluation of the new transmission concept with the optimized transmission ratios is carried out in this chapter. As reference, the powertrain concept that is shown in Figure 9a is chosen. It corresponds to the concept of the Mitsubishi Outlander PHEV. Both of the powertrains have the same ICE and electric motor for traction (the reference requires a second motor for the series hybrid mode). The vehicle dynamics parameterizations of both of the powertrain models are chosen equally and similar to the Mitsubishi Outlander. In order to obtain accurate simulation results, the overall vehicle masses for both powertrain concepts and the power losses within the transmissions are estimated and considered in the powertrain models. The efficiency of each gear pair within the transmissions is chosen to be 0.99 and the drag losses of the clutches and brakes are assumed to be $0.5 \mathrm{Nm}$ (stationary only). The new transmission concept is heavier than the reference concept, but requires only one electric motor. Therefore, the overall mass of the vehicle with the new powertrain concept weighs less than the reference $(\approx 30 \mathrm{~kg})$.

For the comparison of both concepts, DP is applied to determine the optimal control of the powertrain and thus the minimal fuel consumption. The method is carried out with a high resolution and applied to the test cycles-FTP 75, Urban Dynamometer, and WLTP. All of the test cycles are repeated until an overall distance that is equal to approximately $200 \mathrm{~km}$ is driven. Figure $9 \mathrm{c}$ shows the simulated fuel consumptions of the two powertrain models. Depending on the test cycle, the new transmission concept enables fuel savings between $4.4 \%$ and $9.7 \%$. Despite the more complex transmission architecture (higher power losses and weight), fuel savings can be achieved for each driving cycle. The first reason is the increased degree of freedom in terms of the transmission control, 
which enables the ICE and the electric motor to be operated more efficiently. The second reason is the decreased vehicle mass as a result of the usage of only one electric motor. The calculated fuel savings are the theoretical minimum. Considering the real vehicle application, the fuel consumptions will increase, because the simulation does not consider the powertrain dynamics, and the optimal control that is used here is not applicable as the operation modes can oscillate too fast for operating the real transmission. Nevertheless, these investigations provide important information that is required for the development of the new transmission and PHEV concept.

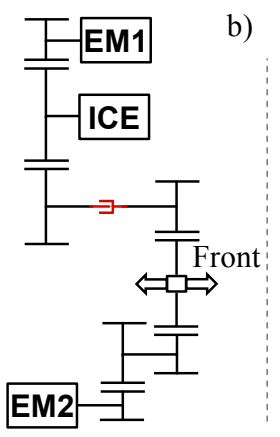

b)

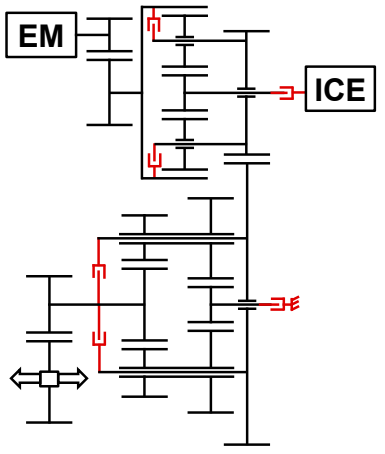

c)

\begin{tabular}{l|c|c|c}
\multirow{2}{*}{ Test Cycle } & $\begin{array}{c}\text { reference } \\
\text { a) }\end{array}$ & $\begin{array}{c}\text { new concept } \\
\text { b) }\end{array}$ & $\begin{array}{c}\text { relative } \\
\text { difference } \\
\text { in \% }\end{array}$ \\
\cline { 2 - 4 } & \multicolumn{2}{|c|}{$\begin{array}{c}\text { fuel consumption } \\
\text { in 1/100km }\end{array}$} & -7.79 \\
\hline FTP 75 & 3.412 & 3.146 & -9.74 \\
\hline $\begin{array}{l}\text { Urban Dyna- } \\
\text { mometer }\end{array}$ & 3.213 & 2.900 & -4.41 \\
\hline WLTP & 3.809 & 3.641 &
\end{tabular}

Figure 9. (a) Reference powertrain concept, (b) powertrain with new transmission concept (see Figure 6), and (c) simulation results, namely: comparison of the fuel consumptions of both powertrains.

\section{Conclusions}

This contribution has described the model-based development and optimization of a new transmission concept for PHEVs. A stationary powertrain model with a vehicle parameterization similar to the Mitsubishi Outlander was used to investigate the benefits of multi-speed transmissions for the ICE and the electric motor. First, the propulsion of the vehicle with the ICE and electric motor was considered separately, in order to estimate the optimal number of speeds. It turned out that a two-speed transmission for the ICE and electric motor are most beneficial. Based on these results, a new transmission concept for PHEVs was developed, which enables an electric driving mode, an ICE mode, a parallel hybrid mode, and a CVT mode. Each mode can be operated in two speeds. By means of the new transmission concept, a further optimization of the transmission ratios was carried out. Here, a combined method of dynamic programming and calculus of variations was applied in order to consider the combined operation of the ICE and electric motor within the optimization. Based on the optimization results, a model-based concept evaluation was carried out. It showed that the new and optimized transmission concept enables a theoretical improvement of $7.3 \%$ fuel saving (in average) compared to a transmission concept with fixed gear ratios for the ICE and electric motor operation.

Author Contributions: Conceptualization, S.G.; Methodology, S.G, A.M. and T.S.; Writing-Original Draft Preparation, S.G.; Writing-Review \& Editing, S.G., A.M. and T.S.; Visualization, S.G. and A.M.; Supervision, T.M.

Funding: This research was funded by the German Federal Ministry of Economics and Energy (BMWi) grant number [01MY13004B].

Acknowledgments: This contribution is accomplished within the project PHEVplus (FKZ: 01MY13004B), funded by the Federal Ministry of Economics and Energy (BMWi) and in cooperation with GKN Driveline International $\mathrm{GmbH}$, www.phevplus.de.

Conflicts of Interest: The authors declare no conflict of interest.

\section{References}

1. Karbowski, D.; Pagerit, S.; Kwon, J.; Rousseau, A.; von Pechmann, K.-F.F. "Fair" Comparison of Powertrain Configurations for Plug-In Hybrid Operation Using Global Optimization; SAE Technical Paper 2009-01-1334; SAE: Detroit, MI, USA, 2009. 
2. Goppelt, G. Das DHT Hybridgetriebe neu definiert. ATZ Automobiltech. Z. 2016, 77, 8-15. [CrossRef]

3. Taniguchi, M.; Yashiro, T.; Takizawa, K.; Baba, S.; Tsuchida, M.; Mizutani, T.; Endo, H. Development of New Hybrid Hybrid Transaxle for Compact-Class Vehicles; SAE Technical Paper, 2016-01-1163; SAE: Detroit, MI, USA, 2016.

4. Conlon, B.M.; Blohm, T.; Harpster, M.; Holmes, A.; Tsuchida, M.; Mizutani, T.; Endo, H.; Kimura, H. The next Generation “Voltec" Extended Range EV Propulsion System. SAE Int. J. Altern. Powertrains 2015, 4, 248-259. [CrossRef]

5. Gassmann, T.; Aikawa, M. GKN Multi-Mode eTransmission for Premium Hybrid Vehicles. In Proceedings of the 12th International CTI Symposium, Berlin, Germany, 2-5 December 2013.

6. Geng, S.; Herber, S.; Hildebrandt, W.; Schulte, T. Powertrain Simulation and Optimization of a Multimode Transmission. In Proceedings of the FISITA World Automotive Congress, Busan, Korea, 26-30 September 2016.

7. Geng, S.; Zubke, T.; Schulte, T. Model-Based Development of Transmission Concepts for Hybrid Electric Powertrains. In Proceedings of the IEEE Intelligent Vehicle Symposium, Redondo Beach, CA, USA, 11-14 June 2017.

8. Silvas, E.; Hofman, T.; Murgovski, N.; Etman, P.; Steinbuch, M. Review of Optimization Strategies for System-Level Design in Hybrid Electric Vehicles. IEEE Trans. Veh. Technol. 2016, 66, 57-70. [CrossRef]

9. Kang, C.; Lee, H.; Kim, J.; Park, Y.; Cha, S. Component Size and Gear Ratio Optimization in PHEV Powertrain. In Proceedings of the IEEE Intelligent Vehicle Symposium, Redondo Beach, CA, USA, 11-14 June 2017.

10. Eghtessad, M. Optimale Antriebsstrangkonfigurationen für Elektrofahrzeuge. Ph.D. Thesis, University of Braunschweig, Braunschweig, Germany, 2014.

11. Murgovski, N.; Johannesson, L.; Sjöberg, J.; Egardt, B. Component Sizing of a Plug-in Hybrid Electric Powertrain via Convex Optimization. Mechatronics 2012, 22, 106-120. [CrossRef]

12. Kim, I.; Kim, H. Configuration Analysis of Plug-in Hybrid Systems using Global Optimization. World Electr. Veh. J. 2013, 6, 391-404. [CrossRef]

13. Herber, S. PHEVplus: Effizienzsteigerndes DHT für Plug-In Hybridanwendungen. In Proceedings of the 17. Internationaler VDI-Kongress Getriebe in Fahrzeugen, Bonn, Germany, 5-6 July 2017.

14. Markel, T.; Brooker, A.; Hendricks, T.; Johnson, V.; Kelly, K.; Kramer, B.; O’Keefe, M.; Sprik, S.; Wipke, K. ADVISOR: A Systems Analysis Tool for Advanced Vehicle Modeling. J. Power Sources 2002, 110, 255-266. [CrossRef]

15. Geng, S.; Schulte, T. Real-Time Powertrain Models of Hybrid Electric Vehicles. SAE Int. J. Altern. Powertrains 2015, 46, 677-682. [CrossRef]

16. Ngo, V.; Hofman, T.; Steinbruch, M.; Serrarens, A. Optimal Control of the Gearshift Command for Hybrid Electric Vehicles. IEEE Trans. Veh. Technol. 2012, 61, 3531-3543. [CrossRef]

17. Naidu, D.S. Optimal Control Systems; Electrical Engineering Textbook Series; CRC Press: Boca Raton, FL, USA, 2003.

18. Kim, N.; Rousseau, A. Sufficient conditions of optimal control based on Pontryagin's minimum principle for use in hybrid electric vehicles. J. Autom. Eng. 2012, 226, 1160-1170. [CrossRef]

19. Bertsekas, D.P. Dynamic Programming and Optimal Control Volume I; Athena Scientific: Belmont, MA, USA, 2005.

(C) 2018 by the authors. Licensee MDPI, Basel, Switzerland. This article is an open access article distributed under the terms and conditions of the Creative Commons Attribution (CC BY) license (http://creativecommons.org/licenses/by/4.0/). 